\title{
Tides of Global Ice-Covered Oceans
}

\author{
Carl Wunsch \\ Department of Earth and Planetary Sciences \\ Harvard University \\ Cambridge MA 02138 \\ email: cwunsch@fas.harvard.edu \\ and \\ Department of Earth, Atmospheric and Planetary Sciences \\ Massachusetts Institute of Technology \\ January 3, 2016
}

\begin{abstract}
The tides of an ice-covered ocean are examined using a Cartesian representation of the elastic and fluid equations. Although unconstrained by any observations, the ocean tides of a Neoproterezoic "snowball" Earth could be significantly larger than they are today. Timemean tidal-residual circulations would then have been set up that are competitive with the circulation driven by geothermal heating. In any realistic configuration, the snowball Earth would have an ice cover that is in the thin shell limit, but by permitting the ice thickness to become large, more interesting ice tidal response can be found, ones conceivably of application to bodies in the outer solar system or hypothetical exoplanets. Little can be said concerning a reduction in tidal dissipation necessary to avoid a crisis in the history of the lunar orbit.
\end{abstract}

\section{Introduction}

Several reasons exist for an exploration of the tides occurring in and under ice sheets, whether floating or land-confined. One motive arises from evidence that approximately 600 million years ago, during the Neoproterezoic, the entire Earth may have frozen, being everywhere covered with ice. Over the ocean, a floating ice sheet may have existed with an estimated thickness of several kilometers (the "hard snowball Earth"). Discussion of the evidence, primarily geological in nature, can be found in Hoffman and Schrag (2002). Ashkenazy et al. (2014; hereafter, A14), describe a theoretical/modelling study of the oceanic circulation that might exist under 
an oceanic ice cover of order of several kilometers. The forcing they assume is purely geothermal, at the average modern rate of roughly $0.1 \mathrm{~W} / \mathrm{m}^{2}$ (Pollack et al., 1993; Davies, 2013), with some localized maxima over ridge-crests. They find an equatorially enhanced meridional overturning circulation, with transports up to $30 \times 10^{6} \mathrm{~m}^{3} / \mathrm{s}$ (30 Sverdrups; Sv) with a nearly homogeneous ocean, both in temperature and salinity. Some account is taken of the oceanic interaction with the overlying ice sheet.

Whether or not a complete snowball Earth actually existed, the question of what the ocean might be like under such circumstances is an interesting theoretical problem. A modern analogue may lie in the outer solar system satellites Enceladus and Europa, which have been inferred to contain fluid oceans covered by multi-kilometer thick ice sheets. In contrast to the A14 solution, discussion of behavior of those oceans has centered on tidal forcing (e.g., Greenberg et al., 1998; Tyler, 2008; Vance and Goodman, 2009; Beuthe, 2015).

Another motivation arises from the known difficulties in accounting for the history of the lunar orbit. The existing rate of tidal dissipation, if constant through time, would have brought the moon catastrophically close to the Earth about 1 billion years ago (e.g., Macdonald, 1964; Goldreich, 1966; Munk, 1967). Munk called the catastrophe the "Gerstenkorn event," and which is known not to have occurred. The conventional interpretation is that lunar tidal dissipation must have been greatly reduced some hundreds of millions of years in the past (see Bills and Ray, 1999 for discussion). Should tidal dissipation have been much reduced during the approximately $200 \mathrm{MY}$ of the Neoproterezoic, it would be a significant contribution to explaining how the reduction occurred. ${ }^{1}$

A comparatively large literature exists on tides induced in ice sheets by the oceanic tidal forcing at the outflow (e.g., Reeh et al., 2003; Thomas, 2007; Arbic et al., 2008). These effects are of at least tangential interest here, but where the focus is instead on the directly driven tidal motions within the ice. Some of the parameter ranges used here are far beyond anything reasonable for the Earth. Perhaps they have some relevance for another planet or satellite.

\footnotetext{
${ }^{1}$ Bartlett and Stevenson (2015) have revived a suggestion of Holmberg (1952), that the principal atmospheric solar semi-diurnal tide - which today effectively accelerates the Earth's rotation - was in a resonant steady-state through much of Earth's history, terminating with the end of the Neoproterezoic. See Munk and Macdonald (1960) for a review of the atmospheric resonance theory, which dates back to Rayleigh's work. Discussion of whether such a high degree of resonance existed and whether it could have persisted through order 1 GY of Earth history is far beyond the scope of the present paper.
} 


\section{A Cartesian Configuration}

Because of all of the uncertainties of the physical setting of the Neoproterezoic Earth, the restricted goals here are to understand the basic physics and to find orders of magnitude of the effects. Only a two-dimensional Cartesian system, as in the Airy "canal theory" of water tides (Lamb, 1932), is used. Consider the situation in Fig. 1, in which an ice sheet of uniform thickness $\bar{d}$ overlies an ocean of constant depth $d$; on the Earth, $d, \bar{d}$ would have an inverse relationship over time. Below the ocean is an infinite elastic half-space. The fluid motion is computed with the half-space not moving, and the ocean tide is computed relative to the sea floor.

Conceptually, as with ocean tides measured from tide gauges, tides within the elastic half-space will produce a modified tidal potential, $U=U_{0}\left(1+k_{L}-h_{L}\right)$, where $U_{0}$ is the gravitational disturbing potential and $k_{L}, h_{L}$ are the conventional Love numbers (Munk and MacDonald, 1960; Lambeck, 1988). The net tide generating potential will be assumed to be,

$$
U=g H e^{i k x-i \sigma t}=g \eta_{E q}
$$

so that the fluid equilibrium height would be $\left|\eta_{E q}\right|=H$, but with the half-space treated here as completely rigid (unmoving).

\subsection{Equations of an Elastic Sheet}

Rheological properties of ice, whether on land or floating, are not simple - encompassing elastic, viscous, plastic and fracture flow laws. MacAyeal and Sergienko (2013) proposed that for timescales of less than about 10 days, treating sea ice as elastic is appropriate and thus reasonable for describing ordinary semi-diurnal or diurnal tides. (Reeh et al., 2003, conclude that incorporation of a land-based part of the glacier would require a viscoelastic rheology, but this bending-beam limit is not undertaken here.) Discussion of long-period tides, including the orbital- rather than rotation-dominated ones in outer-solar system satellites, requires revisiting the rheology and invoking all of viscoelastic, plastic, and fracture mechanisms.

The ice is treated here as purely elastic with Lamé constants $\lambda, \mu$ and the physical and mathematical structure of the problem corresponds to the free-mode analyses of Greenhill (1886), Bromwich (1898), Press and Ewing (1951), and Ewing, Jardetzky and Press (1957, Ch. 5), but in the presence of a periodic body-force of radian frequency $\sigma$. The Cartesian system governing an elastic plate is, 


$$
\begin{aligned}
& \bar{\rho} \frac{\partial^{2} \bar{u}}{\partial t^{2}}=-\sigma^{2} \bar{\rho} \bar{u}=(\lambda+\mu) \frac{\partial}{\partial x}\left(\frac{\partial \bar{u}}{\partial x}+\frac{\partial \bar{w}}{\partial z}\right)+\mu \nabla^{2} \bar{u}+\bar{\rho} \frac{\partial U}{\partial x} \\
& \bar{\rho} \frac{\partial^{2} \bar{w}}{\partial t^{2}}=-\sigma^{2} \bar{\rho} \bar{w}=(\lambda+\mu) \frac{\partial}{\partial z}\left(\frac{\partial \bar{u}}{\partial x}+\frac{\partial \bar{w}}{\partial z}\right)+\mu \nabla^{2} \bar{w}-g \bar{\rho}
\end{aligned}
$$

Variables $\bar{u}, \bar{w}$ in the plate are displacements, not velocities. Barred variables will refer to displacements in the ice layer, unbarred ones to corresponding velocities in the ocean. $\bar{\rho}$ is the density of ice, $U$ has no vertical dependence in the ocean or ice layers and no $y$-dependence is considered. The background gravity $g$ produces a resting static pressure $\bar{\rho} g(\bar{d}-z)$ in the ice; the resulting compaction and induced gravity disturbance are neglected here.

What follows is in the spirit of the paper by Bromwich (1898) and who, as in the papers of Rayleigh and Love, defined the pressure as,

$$
\bar{p}(x, z)=-\lambda\left(\frac{\partial \bar{u}}{\partial x}+\frac{\partial \bar{w}}{\partial z}\right)
$$

taken as finite, but otherwise treated the medium as incompressible with,

$$
\frac{\partial \bar{u}}{\partial x}+\frac{\partial \bar{w}}{\partial z}=0
$$

and implying $\lambda \rightarrow \infty$. One advantage of this system is that it increases the resemblance between the elastic and fluid equations. The sign of $\bar{p}$ has been reversed here from the Bromwich definition, conventional in elasticity, in the interests of that analogy.

In any realistically ice-covered Earth-like ocean, the ice sheet thickness would be a very small fraction of the tidal wavelength, suggesting the use of equilibrium thin-plate theory (e.g., Greenhill, 1886; Landau and Lifschitz, 1970; Turcotte and Schubert, 2002) instead of the dynamical wave equations. That course is not followed so as to make it possible to include the interesting situation in which much thicker ice sheets are disturbed by tides, a configuration perhaps existing in theory in the outer solar system or amongst exoplanets. The "thin-shell" fluid ocean, which gives rise to the Laplace Tidal Equations used here (Cartesian limit), is likely inappropriate for outer solar system satellites, for which a non-hydrostatic, fully spherical coordinate system would be required - as in the Earth's core (Melchior, 1983, Ch. 6; Harrison, 1985).

\subsection{Equations of a Simple Ocean}

Any realistic Earth ocean has a depth, $d$, far less than the tidal forcing wavelength, and a hydrostatic system is adequate, but it proves convenient to consider the more general case. In a non-rotating, constant density, $\rho$, ocean, $-d \leq z \leq 0$, and using the familiar coordinate system 
with $u, w$ being velocities in the positive $x$ and $z$ directions,

$$
\begin{gathered}
-i \sigma \rho u=-\frac{\partial p}{\partial x}+\rho g \frac{\partial U}{\partial x} \\
-i \sigma w=-\frac{\partial p}{\partial z}-g \rho, \\
\frac{\partial u}{\partial x}+\frac{\partial w}{\partial z}=0
\end{gathered}
$$

again linearized about $z=\bar{d}$. Normal stress must vanish there also,

$$
-\bar{p}(\bar{d})+2 \mu \frac{\partial \bar{w}(\bar{d})}{\partial z}+g \bar{\rho} \bar{w}(\bar{d})=0 .
$$




\subsection{Non-dimensional System}

\section{Equations}

With many dimensional quantities defining the system $(d, \bar{d}, \rho, \bar{\rho}, \mu, \sigma, k, g)$, it proves convenient to non-dimensionalize. A system equally useful in both the fluid and elastic media is not, however, obvious. The one chosen here is based upon the conventional time and space scales of ordinary water waves in a homogeneous fluid. Let all primed quantities be non-dimensional and,

$$
\begin{aligned}
T & =1 / \sqrt{g k}, L=1 / k, \\
\bar{U} & =g \bar{\rho} H U^{\prime}, \bar{p}=\bar{\rho} g H \bar{p}^{\prime}, d=L d^{\prime}, \bar{d}=L \bar{d}^{\prime}, \sigma=\sigma^{\prime} \sqrt{g / L}, k^{\prime}=1 \\
(u, w) & =\frac{H}{\sqrt{g / L}}\left(u^{\prime}, w^{\prime}\right), p=\rho g H p^{\prime},(\bar{u}, \bar{w})=H\left(\bar{u}^{\prime}, \bar{w}^{\prime}\right) .
\end{aligned}
$$

The non-dimensional elastic equations become,

$$
\begin{gathered}
\frac{\partial^{2} \bar{u}^{\prime}}{\partial t^{\prime 2}}=-\frac{\partial \bar{p}^{\prime}}{\partial x^{\prime}}+\beta_{1}^{2}\left(\frac{\partial^{2} \bar{u}^{\prime}}{\partial x^{\prime 2}}+\frac{\partial^{2} \bar{u}^{\prime}}{\partial z^{\prime 2}}\right)+\frac{\partial e^{i k^{\prime} x^{\prime}}}{\partial x^{\prime}}, \\
\frac{\partial^{2} \bar{w}^{\prime}}{\partial t^{\prime 2}}=-\frac{\partial \bar{p}^{\prime}}{\partial x^{\prime}}+\beta_{1}^{2}\left(\frac{\partial^{2} \bar{w}^{\prime}}{\partial x^{\prime 2}}+\frac{\partial^{2} \bar{w}^{\prime}}{\partial z^{\prime 2}}\right)-1, \\
\frac{\partial \bar{u}^{\prime}}{\partial x}+\frac{\partial \bar{w}^{\prime}}{\partial z}=0,
\end{gathered}
$$

with $\beta_{1}^{2}=\mu / g \bar{\rho} L=c_{s}^{2} / g L$, and $c_{s}=\sqrt{\mu / \bar{\rho}}$, is the shear wave-speed in the ice, and $\sqrt{g L}=\sqrt{g / k}$ is the ordinary water gravity wave phase speed. Although $k^{\prime}=1$, it is generally displayed as a useful marker.

The corresponding non-dimensional fluid equations are then,

$$
\begin{gathered}
\frac{\partial u^{\prime}}{\partial t^{\prime}}=-\frac{\partial p^{\prime}}{\partial x^{\prime}}+H^{\prime} \frac{\partial e^{i k^{\prime} x^{\prime}}}{\partial x^{\prime}}, \\
\frac{\partial w^{\prime}}{\partial t^{\prime}}=-\frac{\partial p^{\prime}}{\partial z^{\prime}}-1, \\
\frac{\partial u^{\prime}}{\partial x}+\frac{\partial w^{\prime}}{\partial z^{\prime}}=0 .
\end{gathered}
$$

Boundary Conditions 
Non-dimensionalizing as in the equations of motion,

$$
\begin{aligned}
0 & =w^{\prime}\left(-d^{\prime}\right), \\
\bar{w}^{\prime}(0) & =\eta^{\prime} /\left(-i \sigma^{\prime}\right), \\
-\bar{p}^{\prime}(0)+2 \beta_{1}^{2} \frac{\partial \bar{w}^{\prime}(0)}{\partial z^{\prime}}+\bar{w}^{\prime}(0) & =-p^{\prime}, \\
\frac{\partial \bar{u}^{\prime}(\bar{d})}{\partial z^{\prime}}+\frac{\partial \bar{w}^{\prime}(\bar{d})}{\partial x^{\prime}} & =0, \\
-\bar{p}^{\prime}(\bar{d})+2 \beta_{1}^{2} \frac{\partial \bar{w}^{\prime}(\bar{d})}{\partial z^{\prime}}+w^{\prime}(\bar{d}) & =0
\end{aligned}
$$

The simplification $\rho=\bar{\rho}$ has been used. From here on, the primes will be dropped, and unless otherwise stated, all variables are non-dimensional.

\section{Ice Alone}

Consider an elastic ice sheet subject to tidal forcing in which both upper and lower boundaries are free. Absent any $y$-dependence - as is being assumed here - the non-dimensional displacements in the ice can be written generally as,

$$
\bar{u}=\frac{\partial \bar{\varphi}}{\partial x}+\frac{\partial \bar{\psi}}{\partial z}, \bar{w}=\frac{\partial \bar{\varphi}}{\partial z}-\frac{\partial \bar{\psi}}{\partial x}
$$

that is as the gradient of a potential and the curl of a stream function and whose solutions are coupled through the boundary conditions. By Eq. (7c),

$$
\nabla^{2} \bar{\varphi}=0
$$

Eq. (10) is the seismological $P$-(compressional, acoustic) wave equation in the limit as the $P$-wave speed becomes infinite.

Assume all variables are now proportional to $\exp (-i \sigma t+i k x)$. Substituting $\bar{\psi}$ in the two momentum equations, dropping the forcing term, and cross-differentiating to eliminate the pressure produces,

$$
\nabla^{2}\left(\nabla^{2} \bar{\psi}+\frac{\sigma^{2}}{\beta_{1}^{2}} \bar{\psi}\right)=0
$$

or integrating

$$
\left(\nabla^{2} \bar{\psi}+\frac{\sigma^{2}}{\beta_{1}^{2}} \bar{\psi}\right)=M(x, z)
$$

where $M$ is an harmonic function that will be set to zero. The solution then to Eq. (11) is,

$$
\bar{\psi}(x, z)=e^{i k x}\left(A e^{i m z}+B e^{-i m z}\right), m=\sqrt{\frac{\sigma^{2}}{\beta_{1}^{2}}-k^{2}},
$$


and the corresponding velocities are

$$
\bar{u}^{\psi}(x, z)=i m e^{i k x}\left(A e^{i m z}-B e^{-i m z}\right), \bar{w}^{\psi}(x, z)=-i k e^{i k x}\left(A e^{i m z}+B e^{-i m z}\right) .
$$

Substituting back into the homogeneous momentum equations produces $\bar{p}^{\psi}=0, \bar{p}^{\psi}$ being the pressure associated with the stream function.

Let the solution to Eq. (10) be

$$
\bar{\varphi}(x, z)=e^{i k x}\left(C e^{k z}+D e^{-k z}\right)
$$

Substituting $\bar{\varphi}$ into the non-dimensional momentum equations produces,

$$
\begin{aligned}
\frac{\partial^{2}}{\partial t^{2}} \frac{\partial \bar{\varphi}}{\partial x} & =-\frac{\partial \bar{p}}{\partial x}+\frac{\partial e^{i k x}}{\partial x} \\
\frac{\partial^{2}}{\partial t^{2}} \frac{\partial \bar{\varphi}}{\partial z} & =-\frac{\partial \bar{p}}{\partial z}
\end{aligned}
$$

which leads to

$$
\bar{p}(x, z)=e^{i k x}
$$

The boundary conditions are no normal or shear stress on both $z=0, \bar{d}$, and are,

$$
\begin{aligned}
& \left(2 \beta_{1}^{2} k m-i k\right) A+\left(-2 \beta_{1}^{2} k m-i k\right) B+\left(2 \beta_{1}^{2} k^{2}+k\right) C+\left(2 \beta_{1}^{2} k^{2}-k\right) D=1 \\
& \text { no normal stress, } z=0 \\
& \begin{array}{c}
\left(-m^{2}+k^{2}\right) A+\left(-m^{2}+k^{2}\right) B+2 i k^{2} C-2 i k^{2} D=0 \\
\text { no shear stress, } z=0
\end{array} \\
& \left(k^{2}-m^{2}\right) e^{i m \bar{d}} A+\left(k^{2}+m^{2}\right) e^{-i m \bar{d}} B+2 i k^{2} e^{k \bar{d}} C-2 i k^{2} e^{-k \bar{d}} D=0 \\
& \text { no shear stress, } z=\bar{d} \\
& \begin{array}{r}
\left(-2 \beta_{1}^{2} i m k-i k\right) e^{i m \bar{d}} A+\left(2 \beta_{1}^{2} i m k-i k\right) e^{-i m \bar{d}} B+\left(2 \beta_{1}^{2} k^{2}+k\right) e^{k \bar{d}}+\left(2 \beta_{1}^{2} k^{2}-k\right) e^{-k \bar{d}}=1 \\
\text { no normal stress, } z=\bar{d}
\end{array}
\end{aligned}
$$

Taking $\mu=2.3 \times 10^{9}$ (Squire et al., 1995), $\beta_{1}^{2}=0.23$, Fig. 2 shows the response of $2 \mathrm{~km}$ thick ice to a unit amplitude tidal forcing at the $\mathrm{M}_{2}$ period. $\bar{u}, \bar{w}$ and the normal stress are constant with depth in this thin ice sheet, while the shear stress reaches a maximum at mid-depth vanishing as necessary at the two boundaries.

Fig. 3 shows an extreme case of an ice sheet with thickness of the radius of the Earth, and the vertical structure that emerges within the ice from the tidal forcing. This situation could only arise on a hypothetical giant planet.

Much interest exists in the determination of the boundary conditions at the base of continental ice sheets - a generally inaccessible place. The surface response of a continental ice sheet does depend upon those lower boundary conditions and thus the tidal response to a land-fast ice sheet in theory can be used to determine the coupling of ice and land. A useful approach is likely to be the extension to the tidally forced problem of MacAyeal's (1992) ice stream basal stress distribution calculation, but which is not further pursued here. 
Using Eq. (15) to satisfy the boundary condition at $z=-d$, the non-dimensional system of boundary conditions can be written,

$$
\begin{gathered}
-i k A-i k B+k C+k D+\frac{k}{i \sigma} \sinh (k d) E=0, \\
\text { cont. vert. displ., } z=0 \\
\left(\beta_{1}^{2} k m-i k\right) A+\left(-\beta_{1}^{2} k m-i k\right) B+\left(2 \beta_{1}^{2} k^{2}+k\right) C+\left(2 \beta_{1}^{2} k^{2}-k\right) D+ \\
\left(\begin{array}{c}
\left.i \sigma \cosh k d+\frac{k}{i \sigma} \sinh k d\right) E=0, \\
\text { cont. normal stress, } z=0
\end{array}\right. \\
\left(-m^{2}+k^{2}\right) A+\left(-m^{2}+k^{2}\right) B+2 i k^{2} C-2 i k^{2} D+0 E=0, \\
\text { no shear stress, } z=0 \\
\left(2 \beta_{1}^{2} k m-i k\right) e^{i m \bar{d}} A+\left(-2 \beta_{1}^{2} k m-i k\right) e^{-i m \bar{d}} B+\left(2 \beta_{1}^{2}+k\right) e^{k \bar{d}} C+ \\
\text { no shear stress, } z=\bar{d} \\
\left(2 \beta_{1}^{2}-k\right) e^{-k \bar{d}} D+0 E=1 \\
\text { no normal stress, } z=\bar{d}
\end{gathered}
$$


with the last two equations unchanged from those for ice-alone. Setting $\bar{\rho} / \rho=1$ is again a useful approximation in Eq. (20b). In this paper, the frequency is fixed at that corresponding to the $\mathrm{M}_{2}$ tide of $12.42 \mathrm{~h}$ period. Only quantitative change occurs if the frequency is shifted to that of $\mathrm{S}_{1}$ at $24 \mathrm{~h}$ period.

If the response is calculated for an ice sheet of $2200 \mathrm{~m}$ thickness, leaving about $2000 \mathrm{~m}$ of water, the system is near resonance for an ordinary long gravity wave in the ocean alone. Just above resonance, a $-77 \mathrm{~m}$ (inverted tide) is reduced by the ice sheet to $-55 \mathrm{~m}$.

In the limit as $\bar{d} \rightarrow 0$, Eqs. (20e) minus (20a) reduces to the ordinary ocean tide response. Similarly, (20c,d) become identical implying no shear stress in the ice at all. Care must be taken with this numerical limit as the equations become singular.

Fig. 4 shows the response $\eta / \eta_{\text {anal }}$ as a function of $k \bar{d}$ for three values of $d$. The resonance frequency of the ice-free ocean remains essentially unchanged, although the amplitude of the response $\eta$ diminishes, as expected, as the ice thickens, $k \bar{d}$ becoming very large. The resonance appearing when $d$ is dimensionally about $2200 \mathrm{~m}$ is close to that for water alone, when $\sigma=k \sqrt{g d}$. Greenhill (1886) discussed the free periods of a coupled fluid and ice-layer over a rigid half-space, but the values of $k \bar{d}$ at the resonance here is likely beyond the thin plate approximation. On Earth, physically permissible thicknesses leave the ocean tidal response essentially unchanged. However, the oceanic tidal response will generate high wavenumber flows near topography. Because $\bar{d}^{\prime}=k \bar{d}$, Fig. 4 can be interpreted as the reduced response as the ice thickens relative to the wavelength in water and the amplitude suppression becomes significant at short oceanic scales. This impedance change can lead to a reflection of oceanic waves as they approach shallow water, preventing the propagation of energy into highly dissipative shallow regions. Note, however, that the body force in the ocean and ice would then be absent there as a significant contributor.

An Aside - the Laplace Solution

Laplace showed that for a strictly diurnal tide on a water-covered sphere (aquaplanet), $\sigma=\Omega$, $\eta=0,(\Omega$ is the rotation rate of the Earth; see Lamb, 1932, Section 219), that is the oceanic response was a pure oscillating current system with no elevation change, $\eta=0$

$$
\begin{aligned}
& v=-i \frac{g H}{\Omega a} \exp (i \sigma t+i \varphi), \\
& u=\frac{g H}{\Omega a} \cos \theta \exp (i \sigma t+i \varphi),
\end{aligned}
$$

in dimensional form with $\theta, \varphi$ being the colatitude and longitude, with velocities $v, u$ respectively. The solution would imply that the presence of ice was irrelevant (apart from viscous effects). On the other hand, it is not difficult to show that no unforced zero-elevation solution exists: any 
homogeneous solutions required to satisfy a lateral boundary condition would necessarily produce $\eta \neq 0$, and the ice cover would again have to be accounted for.

\section{Snowball Earth-Ocean}

The conclusions from the previous section support the idea that the presence of a global ocean ice cover of thickness of several kilometers does not lead to a significant reduction in tidal amplitudes relative to today. The reduction in water depth, and the likely parallel reduction of tidal motions in highly dissipative shallow water (continental margins) in practice, suggest an increase in tidal amplitudes. For the Earth, the straightforward inference is that ordinary tides of the Neoproterezoic will be at least as large, and probably somewhat larger, than they are today. Near-resonant behavior, such as that seen in some parts of the modern ocean would be expected at that time, but placement, and degree of resonance would all depend sensitively on the continental configuration and the oceanic bottom topography, both of which at the present time are speculative.

The question, raised above, concerning removal of the catastrophic Gerstenkorn event reduction in the lunar orbital radius cannot be addressed without far more detailed information. Indeed, the tidal dissipation rate could conceivably have been even higher under ice. We can however ask order-of-magnitude questions concerning the possible influence of tides in generating a time-mean general circulation in a snowball-Earth-like environment. In the modern ocean, tides are believed to provide a significant fraction of the energy required to sustain the observed three-dimensional circulation (Munk and Wunsch, 1998), roughly about 50\%, with much of the energy used to provide the vertical mixing. Almost all of the rest comes from the wind-fieldassumed absent in an ice-covered world - with some uncertainty remaining from ambiguities of the response to surface buoyancy forcing.

\subsection{Influence on the Circulation}

Consider the energetics of a snowball Earth ocean. The A14 thermal forcing of $0.1 \mathrm{~W} / \mathrm{m}^{2}$ corresponds to a net power input of $3.6 \times 10^{13} \mathrm{~W}$, (36 terrawatts, TW), an impressive amount of energy compared to estimates of the energy required to maintain the modern ocean circulation of roughly $2 \mathrm{TW}$. On the other hand, the A14 solutions depict a circulation with a very small thermal range of about $0.4^{\circ} \mathrm{C}$, and so the Carnot efficiency would be about $0.4 / 273=0.0015$, reducing the usable power to about $50 \mathrm{GW}$. This value is probably an upper bound on the efficiency (e.g., Peixoto and Oort, 1992). Is it possible that the tides of such an ocean would be energetically competitive in sustaining a time-mean flow? Tidal forcing, in contrast, is a direct 
mechanical driver of kinetic energy; but whether a significant large-scale time-mean circulation is generated in practice has to be separated from the question of overall energy input and dissipation.

A considerable literature discusses the rectification of tidal and other oscillatory oceanic flows. Of particular interest in the present context are the papers of Maier-Reimer (1977), Zimmerman (1978), Loder (1980), Huthnance (1981), for homogeneous fluids, and the numerous references found there. Baroclinic flows have been considered by King et al. (2009), Brink (2009), Xing et al. (2011), Grisouard and Bühler (2012), and others. As made particularly clear by Huthnance (1981), the magnitude of time-mean flows depends strongly on details of the nature and magnitude of the dissipation and the topographic configuration. Because large-scale timemean flows will be geostrophically balanced, the inviscid ambiguity of geostrophy (Moore, 1970) has to be properly accounted for via the frictional (or other) mechanisms; see for example, Visser (1994).

Any discussion of the tide-induced mean circulation in the Neoproterezoic ocean is necessarily pure speculation, and no detailed calculation seems justified. An order of magnitude estimate is perhaps reasonable. Here, following Huthnance (1981) and ignoring any interaction with the flow driven geothermally as in A14, his equations 5.3, 5.4 for one-dimensional analysis gives rise to a geostrophic mean flow, with linear friction at the sea bottom and/or at the water-ice boundary,

$$
\langle v\rangle \approx-2 \Omega \cos \theta\left\langle\left(\frac{v_{\text {tide }}}{\sigma}\right)^{2}\right\rangle \frac{1}{d} \frac{\partial d}{\partial x}
$$

Here $\theta$ is again the colatitude, and $x$ is any coordinate perpendicular to the topographic contours, where $d=d(x)$. A coast is supposed to exist to support the geostrophic pressure gradient (but see Huthnance's Eq. 5.5). $v_{\text {tide }} / \sigma$ is the magnitude of the horizontal excursion in the oscillating tide. For an order of magnitude, suppose the excursion is about $100 \mathrm{~m}$ (a bit less than implied by Eqs. (21)), that the latitude is $30^{\circ}$, and that the slope, $1 / d \partial d / \partial x=0.01$. Then $\langle v\rangle \approx 0.01$ $\mathrm{m} / \mathrm{sec}$. A barotropic flow of this magnitude in $4000 \mathrm{~m}$ of water produces a transport of about $40 \mathrm{~m}^{2} / \mathrm{s}$. In a section half-spanning the Pacific Ocean (circa 5,000 km) the meridional transport is about $200 \mathrm{~Sv}$ as compared to $30 \mathrm{~Sv}$ zonal maximum near the equator estimated by A14 for the geothermal response. Lots of scope is available in Eq. (22) for changing this value, both upwards and downwards, including the change with colatitude, focussing on shallow water, regions of steep slope etc. But the subject is left here as apparently being an observationally untestable one. 


\subsection{More Realism}

Tides of the modern realistic ocean are considerably more complex, involving rotation, interaction with boundaries, dissipation, topography, and stratification. Some properties even of the non-rotating canal theory do remain robust in the presence of all of these complications as the barotropic solutions (no stratification) remain governed by gravity-wave physics even where rotation is important. Near-resonances still appear, although they can also be generated by the presence of sidewalls and not just from travelling-wave version seen here; dissipation and nonlinearity enter. Particle velocities are strongly influenced by rotation, as would the boundary-layer between the ocean and the base of the ice sheet. (Hendershott, 1981, is a useful review of the physics of modern ocean tides.)

In a non-rotating ocean, an important mechanical boundary layer scale would be $(A / \sigma)^{1 / 2}$, becoming with finite rotation, $\left(A / \sqrt{\sigma^{2}-f^{2}}\right)^{1 / 2}$, where $f=2 \Omega \sin \phi$, and $\phi$ the latitude and $A$ is a hypothetical eddy-viscosity. At latitudes where $\sigma \approx f$ (the "inertial latitude"), the boundary layer physics are distinct. For semi-diurnal tide constituents, that occurs only poleward of about $70^{\circ}$ latitude, but for diurnals it is at about $30^{\circ}$.

Numerous studies exist of the boundary layer flows under ice in the Arctic (e.g., McPhee, 2002; Cole et al., 2014) where rotation tends to be important or dominant. For obvious reasons, no modern observations exist of low-latitude, small $f$, sea ice-ocean boundary layer interactions. Under-ice topography can be very rough, and how to model the fluid interactions at low latitudes is also not so clear. A reasonable inference is that dissipation at the sea ice-water boundary would be at least as important as tidal dissipation over the bottom on abyssal plains today, and probably considerably greater.

Of central concern in discussing a snowball Earth is the topographic change: modern-day tides have a substantial fraction of their dissipation occurring in the shallow regions of the continental margins (Egbert and Ray, 2001). Tidal response in shallow water, $d^{\prime}<<d$, is largely a "co-oscillation" forced by the incoming tide from deeper water, instead of being a direct response to the local forcing. In an ice-covered ocean with shallow margins, the deep water tide would tend to undergo reflection as the ice-lid becomes ever-more effective with vanishing $d^{\prime} / \bar{d}$, and it is a reasonable surmise that continental margin dissipation would be greatly reduced relative to today's values (Fig. 4).

The second major tidal dissipation mechanism in the modern ocean is through the baroclinic conversion from the stratification and the presence of topography (e.g., Egbert and Ray, 2000). This physics requires a stratified fluid, and as the A14 ocean is nearly unstratified, the role of tidal mixing is far from obvious. If the snowball ocean is nearly unstratified, baroclinic 
conversion would also be much reduced. Thus both major dissipation mechanisms may have been weaker, and the perhaps paradoxical inference is that tides of an ocean with an ice-lid are potentially considerably stronger than they are today. An important caveat is that proximity to resonance is a sensitive function of the continental configuration and which will have changed greatly through millions of years.

\section{Other Processes}

None of the present results as applied to the snowball Earth are definitive, and many unknowns and complications intervene. Among other intriguing complications not discussed here are the role of the changed Earth rotation rate and length of the month at times approaching -1 GY when the day was probably about 22 hours long, and with about 13 synodic months in the year (Bills and Ray, 1999; Williams, 2000). These changes are consequences of tidal friction and the resulting braking of Earth-spin over time.

If the modern ocean depth is reduced by half, and assuming that 600 million years ago that the salt amount in the ocean was similar to today, salinity would have roughly doubled to about $7 \%$ of the water mass. A salty fluid, heated from below, can be unstable to double-diffusive processes (see e.g., Turner, 1973; Brandt and Fernando, 1995; Radko, 2013) forming a layered circulation. Whether over millions of years that possibility persists, and what would be the consequences of any annual cycling at low latitudes in the ice-cover, has not been discussed. If some stratification does persist, then baroclinic tidal conversion can occur, a spatially dependent mixing would arise, and a whole suite of further theoretical problems can be defined including the baroclinic mean flows already alluded to above. Because observational tests of solutions in such interesting configurations appear safely unlikely, we leave the problem here as one of near-total speculation. The outer solar system bodies may eventually become more accessible to observation than is the Neoproterezoic ocean.

Considerable debate exists about the possibility that the near-equatorial ocean remained icefree during the Neoproterezoic - much of the argument concerning the ability of multi-celled life to survive a complete hard snowball (e.g., Hyde et al., 2000). Determining the tidal response in such a configuration represents another potentially interesting theoretical calculation probably not justified at present. 


\section{Summary Comments}

From "canal-theory"-like calculations, the ordinary semi-diurnal and diurnal tides of a snowballEarth are found likely to be at least as strong as those in the modern ocean. As with modern tides, details depend sensitively on bottom topography, continental configurations, dissipation mechanisms, and overall water depths. Consequently, tidal motions, and in particular rectified mean values, are not obviously negligible in the discussion of the oceanic general circulation at that time. Similarly, no conclusion can be drawn about changes in tidal dissipation with its consequences for the history of the lunar orbit.

In extending the present results to outer solar system satellites and exoplanets, the basic methodology can be maintained. Changes are required for the rheology of ice (even if dominantly water-ice, viscoelastic, plastic and fracture mechanisms can come into play). For satellites or planets locked in spin-orbit coupling, the long-period tides will dominate rather than the semidiurnal or diurnal components focussed on here. The thin-shell limit of the Laplace Tidal Equations can also become too inaccurate.

The possibility of paleo-observations shedding light on the details of the tides in the Earth's history appears remote. Oceans on outer satellites of the solar system or on exoplanets may in the long-term be more observationally accessible.

Acknowledgements J. Rice and E. Tziperman provided useful comments on an early version of the manuscript. Supported in part by the National Science Foundation under Grant OCE0961713 at MIT. 
Arbic, B. K., Mitrovica, J. X., MacAyeal, D. R., Milne, G. A., 2008. On the factors behind large Labrador Sea tides during the last glacial cycle and the potential implications for Heinrich events. Paleoceanog.. 23, PA3211.

Ashkenazy, Y., Gildor, H., Losch, M., Tziperman, E., 2014. Ocean circulation under globally glaciated snowball Earth conditions: Steady-state solutions. J. Phys. Oc.. 44, 24-43.

Bartlett, B. C., Stevenson, D. J., 2015. Analysis of a Precambrian resonance-stabilized day length. arXiv:1502.01421 [physics.geo-ph].

Beuthe, M., 2015. Tides on Europa: The membrane paradigm. Icarus. 248, 109-134.

Bills, B. G., Ray, R. D., 1999. Lunar orbital evolution: A synthesis of recent results. Geophys. Res. Letts. 26, 3045-3048.

Brandt, A., Fernando, H. J. S., Eds. 1995. Double-Diffusive Convection. Geophysical Monog. 94. Am. Geophys. Un., Washington.

Brink, K. H., 2011. Topographic rectification in a stratified ocean. J. Mar. Res. 69, 483-499. Bromwich, T. I. A., 1898. On the influence of gravity on elastic waves, and, in particular on the vibrations of an elastic globe. Proc. London Math. Soc. 1, 98-165.

Cole, S. T., Timmermans, M. L., Toole, J. M., Krishfield, R. A., Thwaites, F. T., 2014. Ekman veering, internal waves, and turbulence observed under Arctic Sea ice. J. Phys. Oc.. 44, 13061328 .

Davies, J. H., 2013. Global map of solid Earth surface heat flow. Geochem. Geophys. Geosyst. $14,4608-4622$.

Egbert, G. D., Ray, R. D., 2000. Significant dissipation of tidal energy in the deep ocean inferred from satellite altimeter data. Nature. 405, 775-778.

Egbert, G. D., Ray, R. D., 2001. Estimates of M-2 tidal energy dissipation from TOPEX/Poseidon altimeter data. J. Geophys. Res.-Oceans. 106, 22475-22502.

Ewing, W. M., Jardetzky, W. S., Press, F., 1957. Elastic Waves in Layered Media. McGrawHill, New York.

Goldreich, P., 1966. History of the lunar orbit. Revs. Geophys. 4, 411-439.

Greenberg, R., et al., 1998. Tectonic processes on Europa: Tidal stresses, mechanical response, and visible features. Icarus. 135, 64-78.

Greenhill, A. G., 1886. Wave motion in hydrodynamics. Am. J. Maths. 9, 62-96.

Grisouard, N., Bühler, O., 2012. Forcing of oceanic mean flows by dissipating internal tides. J.

Fluid Mech.. 708, 250-278.

Harrison, J. C., 1985. Earth Tides. Van Nostrand Reinhold Co., New York. 
Hendershott, M., 1981. Long waves and ocean tides. In Evolution of Physical Oceanog., B. A. Warren and C. Wunsch, Eds., The MIT Press, Cambridge, 292-341.

Hoffman, P. F., Schrag, D. P., 2002. The snowball Earth hypothesis: testing the limits of global change. Terra Nova. 14, 129-155.

Holmberg, E. R. R., 1952, A suggested explanation of the present value of the velocity of rotation of the Earth. Month. Not. Roy. Astron. Soc., 112, 325-330.

Huthnance, J. M. On mass transports generated by tides and long waves. J. Fluid Mech. 102, 367-387.

Hyde, W. T., Crowley, T. J., Baum, S. K., Peltier, W. R., 2000. Neoproterezoic 'snowball Earth' simulations with a coupled climate/ice-sheet model. Nature 405, 425-429.

King, B., Zhang, H. P., Swinney, H. L., 2009. Tidal flow over three-dimensional topography in a stratified fluid. Physics of Fluids. 21, 116601-10.

Lamb, H., 1932. Hydrodynamics, Sixth ed. Dover, New York.

Lambeck, K., 1988. Geophysical Geodesy: The Slow Deformations of the Earth. Oxford University Press, Oxford England, New York.

Landau, L. D., Lifshits, E. M., 1970. Theory of Elasticity. Pergamon Press, Oxford, New York,. Loder, J. W., 1980. Topographic rectification of tidal current on the sides of Georges Bank. J. Phys. Oc.. 10, 1399-1416.

MacAyeal, D. R., 1992. The basal stress distribution of Ice Stream E, Antarctica, inferred by control methods. J. Geophys. Res. Solid Earth. 97, 595-603.

MacAyeal, D. R., Sergienko, O. V., 2013. The flexural dynamics of melting ice shelves. Ann. Glaciol. 54, 1-10.

Macdonald, G. J. F., 1964. Tidal friction. Revs. Geophys. 2, 467-541.

Maier-Reimer, E., 1977. Residual circulation in the North Sea due to the M2-tide and mean annual wind stress. Deut. Hydrograph. Zeit., 30,69-80.

McPhee, M. G., 2002. Turbulent stress at the ice/ocean interface and bottom surface hydraulic roughness during the SHEBA drift. J. Geophys. Res.-Oceans. 107 DOI: 8037 10.1029/2000jc000633.

Melchior, P. J., 1983. The Tides of the Planet Earth. Pergamon Press, Oxford, New York.

Moore, D., 1970. The mass transport velocity induced by free oscillations at a single frequency.

Geophys. Fl. Dyn. 1, 237-247.

Munk, W., 1968. Once again-tidal friction. Q. J. Roy. Astron. Soc., 9. 352-375.

Munk, W., Wunsch, C., 1998. Abyssal recipes II: energetics of tidal and wind mixing. Deep-Sea Res., 45. 1976-2009.

Munk, W. H., MacDonald, G. J. F., 1960. The Rotation of the Earth: A Geophysical Discussion. Cambridge University Press, Cambridge. 
Peixoto, J. P., Oort, A. H., 1992. Physics of Climate. Amer. Inst. Phys., New York.

Pollack, H. N., Hurtrer, S. J., Johnson, J.R., 1993. Heat flow from the Earth's interior: analysis of the global data set. Revs. Geophys. 31, 267-280.

Press, F. and Ewing, M., 1951. Propagation of elastic waves in a floating ice sheet. Trans. Am. Geophys. Un., 32, 673-678.

Radko, T., 2013. Double Diffusive Convection. Cambridge Un. Press, Cambridge.

Reeh, N., Christensen, E. L., Mayer, C., Olesen, E. B., 2003. Tidal bending of glaciers: a linear viscoelastic approach. Ann. Glaciol. 37, 83-89.

Squire, V. A., Dugan, J. P., Wadhams, P., Rottier, P. J., Liu, A. K., 1995. Of ocean waves and sea-ice. Ann. Rev. Fluid Mechs. 27, 115-168.

Thomas, R. H., 2007. Tide-induced perturbations of glacier velocities. Global Planet. Change. $59,217-224$.

Turcotte, D. L., Schubert, G., 2002. Geodynamics, 2nd Ed. Wiley, New York.

Turner, J. S., 1973. Buoyancy Effects in Fluids. Cambridge Un. Press, Cambridge.

Tyler, R. H., 2008. Strong ocean tidal flow and heating on moons of the outer planets. Nature. 456, 770-U55.

Vance, S., Goodman, J., 2009. Oceanography of an ice-covered moon. Europa, edited by RT Pappalardo, WB McKinnon, and K. K Khurana, University of Arizona Press, Tucson, AZ. 459482 .

Visser, A. W., 1994. On tidal rectification, friction, and geostrophic rectification. J. Phys. Oc.. 24, 2196-2200.

Williams, G. E., 2000. Geological constraints on the Precambrian history of Earth's rotation and the Moon's orbit. Revs. Geophys. 38, 37-59.

Xing, J. X., Davies, A. M., Jones, J. E., 2011. Application of an unstructured mesh model to the determination of the baroclinic circulation of the Irish Sea. J. Geophys. Res.-Oceans. 116.

Zimmerman, J. T. F., 1978. Topographic generation of residual circulation by oscillatory (tidal) currents. Geophys. Astrophys. Fl. Dyn. 11, 35-47. 
1. Defining geometry of an ice layer of thickness $\bar{d}$ over an ocean of depth $d$. Below the ocean is an infinite, rigid, half-space. $z$ is directed vertically upward from the ice-ocean interface.

2. Solution as a function of non-dimensional $k z$ for an ice sheet of thickness $\bar{d}=2000 \mathrm{~m}$, with free-free boundaries subject to a unit amplitude body tide at the $\mathrm{M}_{2}$ frequency. $\operatorname{Re}(\bar{u}), \operatorname{Im}(\bar{w})$ are shown (b), as well (a) as the shear stress as a function of depth, and the normal stress (c). Depth is normalized by $k$.

3. Same as Fig. 2 except for $\bar{d}=2 \pi / k$, the radius of the Earth.

4. (Lower panel) $\eta$ as a function of $k \bar{d}$ for the combined ocean-ice case, for 3 different values of $d$ at the $\mathrm{M}_{2}$ tidal frequency. (Upper panel ) $\eta / \eta_{\text {equil }}$ as a function of $k \bar{d}$ for three different values of $d$. Largest value of $\bar{d}$ corresponds to a dimensional ice thickness of 200,000 m. 


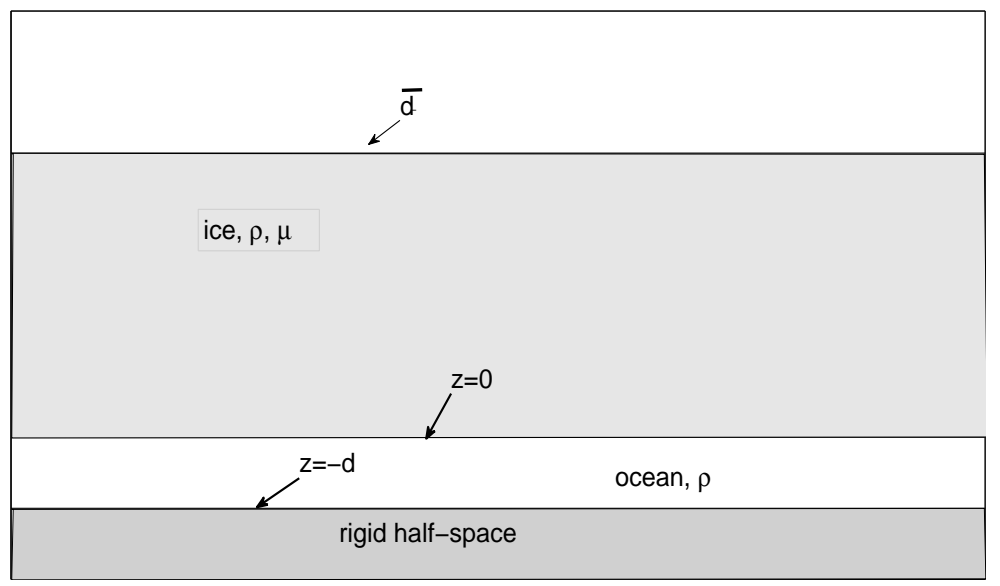

Figure 1: Defining geometry of an ice layer of thickness $\bar{d}$ over an ocean of depth $d$. Below the ocean is an infinite, rigid, half-space. $z$ is directed vertically upward from the ice-ocean interface.
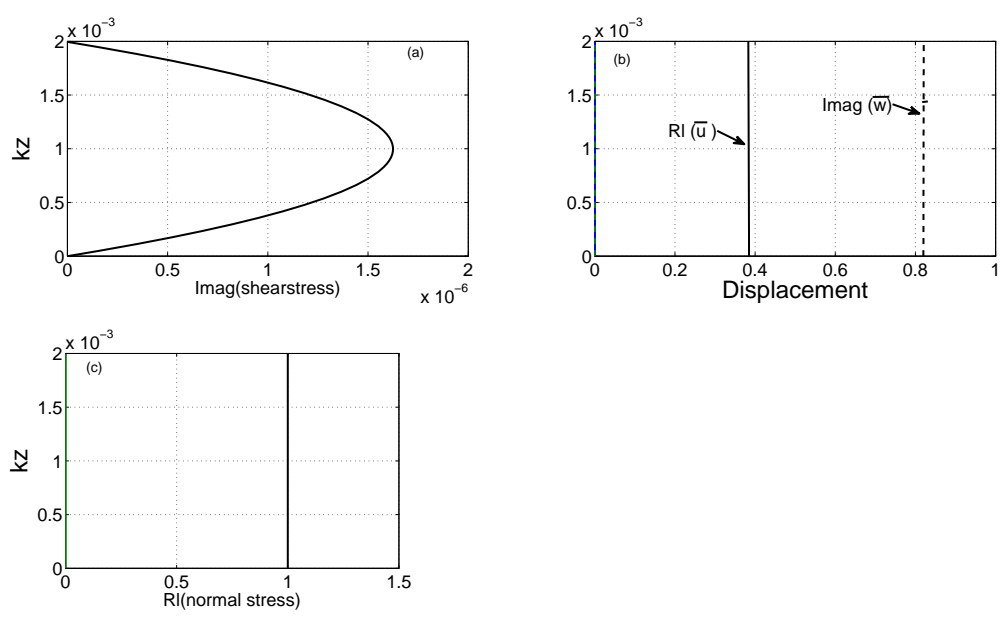

Figure 2: Solution as a function of non-dimensional $k z$ for an ice sheet of thickness $\bar{d}=2000 \mathrm{~m}$, with free-free boundaries subject to a unit amplitude body tide at the $\mathrm{M}_{2}$ frequency. $\operatorname{Re}(\bar{u}), \operatorname{Im}(\bar{w})$ are shown (b), as well (a) as the shear stress as a function of depth, and the normal stress (c). Depth is normalized by $k$. 

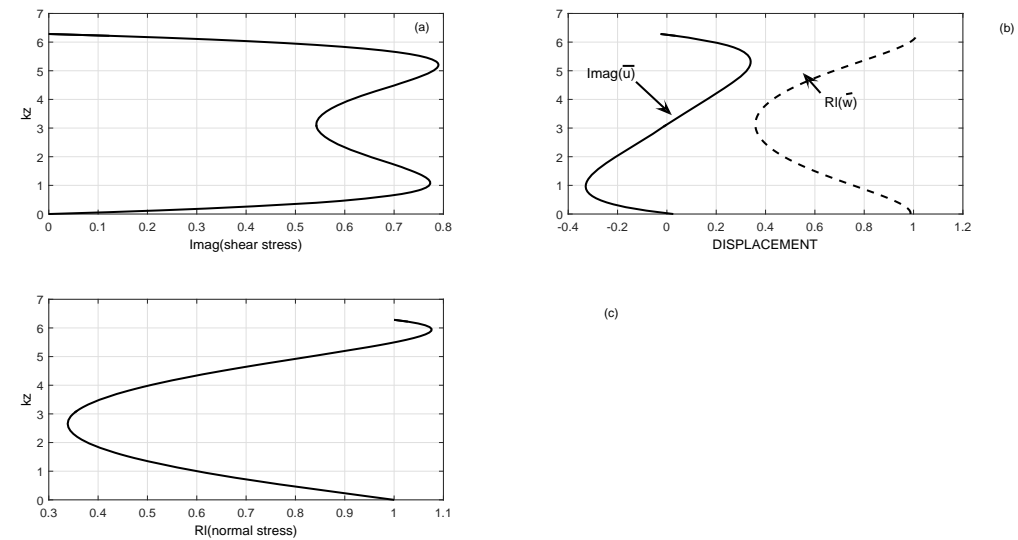

(c)

Figure 3: Same as Fig. 2 except for $\bar{d}=2 \pi / k$, the radius of the Earth.
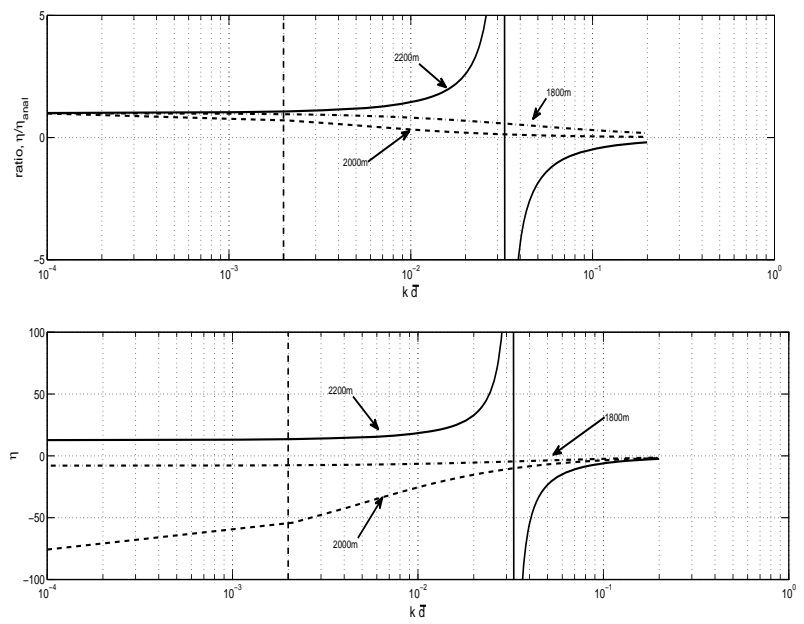

Figure 4: (Lower panel) $\eta$ as a function of $k \bar{d}$ for the combined ocean-ice case, for 3 different values of $d$ at the $\mathrm{M}_{2}$ tidal frequency. (Upper panel ) $\eta / \eta_{\text {equil }}$ as a function of $k \bar{d}$ for three different values of $d$. Largest value of $\bar{d}$ corresponds to a dimensional ice thickness of $200,000 \mathrm{~m}$. 